EPJ Web of Conferences 66, 05005 (2014)

DOI: $10.1051 /$ epjconf/ 20146605005

(C) Owned by the authors, published by EDP Sciences, 2014

\title{
Active nuclear spin maser oscillation with double cell
}

E. Hikota ${ }^{1}$,a, M. Chikamori ${ }^{1}$, Y. Ichikawa ${ }^{1}$, Y. Ohtomo ${ }^{1}$, Y. Sakamoto ${ }^{1}$, T. Suzuki ${ }^{1}$, C.P. Bidinosti ${ }^{2}$, T. Inoue ${ }^{3}$, T. Furukawa ${ }^{4}$, A. Yoshimi ${ }^{5}, \mathrm{~K}_{\text {. Suzuki }}{ }^{1}$, T. Nanao ${ }^{1}$, H. Miyatake ${ }^{1}$, M. Tsuchiya ${ }^{1}$, N. Yoshida ${ }^{1}$, H. Shirai ${ }^{1}$, T. Ino ${ }^{6}$, H. Ueno ${ }^{7}$, Y. Matsuo ${ }^{7}$, T. Fukuyama ${ }^{8}$, and K. Asahi ${ }^{1}$

\footnotetext{
${ }^{1}$ Department of Physics, Tokyo Institute of Technology, 2-12-1 Oh-okayama, Meguro, Tokyo 152-8551, Japan

${ }^{2}$ Department of Physics, University of Winnipeg, 515 Portage Avenue, Winnipeg, Manitoba, Canada

${ }^{3}$ Cyclotron and Radioisotope Center, Tohoku University, 6-3 Aoba, Aramaki, Aoba, Sendai 980-8578, Japan

${ }^{4}$ Department of Physics, Tokyo Metropolitan University, 1-1 Minami-Ohsawa, Hachioji, Tokyo 192-0397, Japan

${ }^{5}$ Research Core for Extreme Quantum World, Okayama University, 3-1-1 Tsushimanaka, Kita, Okayama 700-8530, Japan

${ }^{6}$ Institute of Material Structure Science, High Energy Accelerator Research Organization (KEK), 1-1 Oho, Tsukuba, Ibaraki 305-0801, Japan

${ }^{7}$ RIKEN Nishina Center, 2-1 Hirosawa, Wako, Saitama 351-0198, Japan

${ }^{8}$ R-GIRO, Ritsumeikan University, 1-1-1 Noji-Higashi, Kusatsu, Shiga 525-8577, Japan
}

\begin{abstract}
Uncertainty in the frequency precision of the planned experiment to search for $\mathrm{a}^{129} \mathrm{Xe}$ atomic electric dipole moment is dominated by drifts in the frequency shift due to contact interaction of ${ }^{129} \mathrm{Xe}$ with polarized $\mathrm{Rb}$ valence electrons. In order to suppress the frequency shift, a double-cell geometry has been adopted for the confinement of ${ }^{129} \mathrm{Xe}$ gas. A new process has been identified to take part in the optical detection of spin precession. The parameters controlling the oscillation of the maser in this new double-cell arrangement were optimized. As a result, the frequency shift has been reduced by a factor of 10 or more from the former single-cell geometry.
\end{abstract}

\section{Introduction}

A permanent electric dipole moment (EDM) which directly violates time reversal symmetry attracts much attention, because an unknown CP-violating phase which is necessary to understand the present matter-dominated Universe is expected to be probed by EDM. The present study aims at measuring the EDM in the diamagnetic atom ${ }^{129} \mathrm{Xe}$ to the order of $|d|=10^{-28} \mathrm{ecm}$, beyond the present upper limit [1]. In the EDM measurement, we employ an active nuclear spin maser [2, 3] which enables us to sustain the spin precession of ${ }^{129} \mathrm{Xe}$ over a long measurement duration. The spin precession of ${ }^{129} \mathrm{Xe}$ is detected optically through transverse polarization of $\mathrm{Rb}$ spins. An oscillating magnetic field is applied in synchronization with the spin precession, thereby preventing transverse spin relaxation. Previous development on the active spin maser has achieved a precision $\delta v=9.3 \mathrm{nHz}$ for the frequency averaged over a single-shot observation of continued precession sustained for $3 \times 10^{4} \mathrm{~s} \mathrm{[3]}$.

\footnotetext{
ae-mail: hikoeri@th.phys.titech.ac.jp
} 


\section{EPJ Web of Conferences}

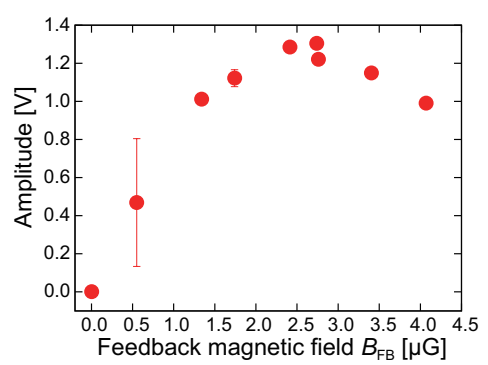

Figure 1. Optimization of the feedback magnetic field $B_{\mathrm{FB}}$. The amplitude of the maser oscillation is plotted as a function of $B_{\mathrm{FB}}$. In this measurement, temperature was kept at $\sim 70{ }^{\circ} \mathrm{C}$. The maximum amplitude was obtained with a feedback field of $B_{\mathrm{FB}}^{\max }=2.5 \mu \mathrm{G}$.

It is important to note, however, that the above frequency precision will be meaningful to an EDM detection only if the magnetic field acting on the spins is stable, or if its average in the same time period is known, to an equal or higher level of precision. A comagnetometer using ${ }^{3} \mathrm{He}$ was incorporated into the active spin maser system in order to cancel out the drifts in the external magnetic field $B_{0}$ which are commonly sensed by ${ }^{129} \mathrm{Xe}$ and ${ }^{3} \mathrm{He}$ [4]. However the drifts in the frequency shift due to contact interaction of ${ }^{129} \mathrm{Xe}$ spin with polarized $\mathrm{Rb}$ valence electrons can not be fully removed by the ${ }^{3} \mathrm{He}$ co-magnetometer, because of the different strengths for the $\mathrm{Rb}-{ }^{129} \mathrm{Xe}$ and $\mathrm{Rb}-{ }^{3} \mathrm{He}$ contacts [5, 6]. Therefore a double-cell geometry [1] in which the gas volume is divided into one section for the optical pumping and one section for masing and optical spin detection was employed. The magnitude of the frequency shift is proportional to the product of the $\mathrm{Rb}$ polarization and the $\mathrm{Rb}$ number density given as a function of the temperature. The double-cell geometry enables us to suppress $\mathrm{Rb}$ polarization in the optical detection part and thus to reduce the frequency shift.

\section{Performance estimation with the single-cell maser}

We first evaluate the magnitude of the frequency shift using a cell in which the pumping section and the detection section are merged into one single spherical cell, containing ${ }^{129} \mathrm{Xe}$ and $\mathrm{N}_{2}$ gases at 230 Torr and 100 Torr, respectively. The experimental setup is the same as in Ref. [4] except for the cell. The precession frequency of the free induction decay (FID) was measured for several different values of the static magnetic field $B_{0}$, and the intercept at $B_{0}=0$ was evaluated. The same procedure was followed after the circular polarization of the pumping laser light was reversed. In order to cancel out the residual magnetic field in the shield, the frequency shift was determined by averaging the two intercepts, to be $90 \mathrm{mHz}$ and $22 \mathrm{mHz}$ at $100{ }^{\circ} \mathrm{C}$ and $75^{\circ} \mathrm{C}$, respectively.

We next seek the optimum condition for the maser oscillation. The amplitude of the maser oscillation of ${ }^{129} \mathrm{Xe}$ was recorded by varying the strength of the feedback magnetic field $B_{\mathrm{FB}}$, as shown in Fig. 1. The maximum amplitude was obtained with a feedback field of $B_{\mathrm{FB}}^{\max }=2.5 \mu \mathrm{G}$. According to the modified Bloch equations describing the dynamics of the maser [2], the optimum feedback field $B_{\mathrm{FB}}^{\max }$ yielding the maximum amplitude is written as $B_{\mathrm{FB}}^{\max }=\gamma^{-1}\left(T_{1}^{*} T_{2}\right)^{-1 / 2}$, where $\gamma$ is the gyromagnetic ratio of ${ }^{129} \mathrm{Xe}, T_{1}^{*}$ the effective longitudinal relaxation time, and $T_{2}$ the transverse relaxation time. Under the above optimum condition, $T_{1}^{*}$ and $T_{2}$ were evaluated to be $89.2 \mathrm{~s}$ and $34.0 \mathrm{~s}$, respectively, giving $B_{\mathrm{FB}}^{\max }=2.46 \mu \mathrm{G}$, which agrees well with the above optimum field obtained empirically. Thus, the optimum condition for the maser oscillation has been quantitatively established.

In the operation of the active spin maser with the double-cell geometry, there may arise two difficulties. One is the reduction of ${ }^{129} \mathrm{Xe}$ polarization due to gas diffusion. The inner surface of the cell was coated with SurfaSil, in order to suppress the spin relaxation at the inner wall and to allow the ${ }^{129} \mathrm{Xe}$ atoms to pass through the connecting tube without loss of polarization. Another difficulty is a possible deterioration of the maser oscillation signal due to reduced $\mathrm{Rb}$ polarization. 

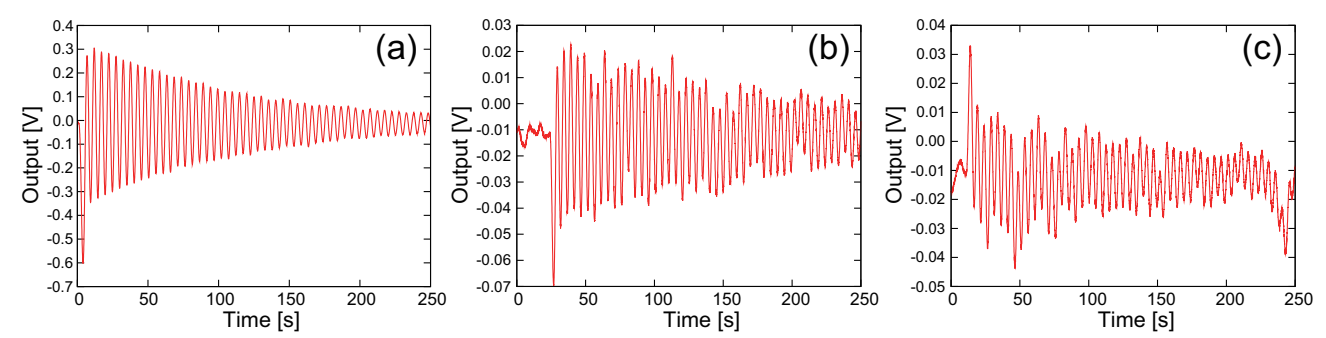

Figure 2. Free induction decays indicating the transverse component of precessing ${ }^{129} \mathrm{Xe}$ spins: (a) with circularly polarized pumping light incident on the cell, (b) with the pumping laser turned off before application of the rf tipping pulse, and (c) with linearly polarized pumping light incident on the cell.

In order to reveal the effect due to reduction of the $\mathrm{Rb}$ polarization, three FID measurements were conducted with the same single cell, where conditions for the pumping laser light were changed. First, a pumping laser light which was circularly polarized was incident on the cell, and the FID signal was recorded after applying an rf field pulse. Second, the FID signal was taken with the pumping laser turned off just before applying the rf field, a situation equivalent to the case with the double-cell geometry. Third, the measurement was conducted again but this time with a linearly polarized laser light in order to maintain the cell temperature unaltered from the first measurement. Results of the three experiments are presented in Fig. 2. These results reveal that there are two processes responsible for the optical detection, one dependent and the other independent of the Rb polarization.

Previously, we studied the performance of an active spin maser with a single cell. The spin detection there relied on the following mechanism: the transverse spin polarization of ${ }^{129} \mathrm{Xe}$ is transferred to $\mathrm{Rb}$ atoms via spin exchange (repolarization) [3], and letting the $\mathrm{Rb}$ transverse spins follow the ${ }^{129} \mathrm{Xe}$ precession. Thus, the ${ }^{129} \mathrm{Xe}$ precession is detected through a change in the transmission of circularly polarized light through the cell perpendicular to the $B_{0}$ field. This repolarization process is independent of the $\mathrm{Rb}$ polarization. The above results, however, imply that there actually exists another process which depends on the Rb polarization. At present, we tentatively consider that the new process proceeds as follows: the polarization axis for $\mathrm{Rb}$ atoms follows adiabatically the instantaneous direction of the magnetic field. Since the field acting on the cell is a vector sum of the applied field $B_{0}$ and the dipolar field due to the polarized ${ }^{129} \mathrm{Xe}$ spin, the $\mathrm{Rb}$ polarization is pointed in a direction slightly tilted from $B_{0}$ and precessing with the ${ }^{129} \mathrm{Xe}$ magnetization. Thus the transverse component of the $\mathrm{Rb}$ polarization is synchronized with the ${ }^{129} \mathrm{Xe}$ precession, so this may contribute to the optical spin detection mechanism. The magnitude of this signal should be proportional to the $\mathrm{Rb}$ polarization. This component should have been contributing in the single-cell geometry, but in the maser operation with the double-cell geometry, this process tends to be hindered because the Rb polarization is small, and only the repolarization process will be effective. Taking account of the three FID experiments and the two processes, the signal amplitude from the active spin maser with the double-cell geometry is estimated to be $\sim 1 / 7$ of that with the single-cell geometry, provided that the possible reduction in ${ }^{129} \mathrm{Xe}$ polarization due to diffusion is not taken into account.

\section{Performance test of the maser with double-cell geometry}

Bearing the above estimation in mind, performance of the active spin maser with the double-cell geometry was tested. The experimental setup was the same as before but the single cell was replaced with the double cell, containing 10 Torr of ${ }^{129} \mathrm{Xe}, 470$ Torr of ${ }^{3} \mathrm{He}$ and 100 Torr of $\mathrm{N}_{2}$ gases. The 


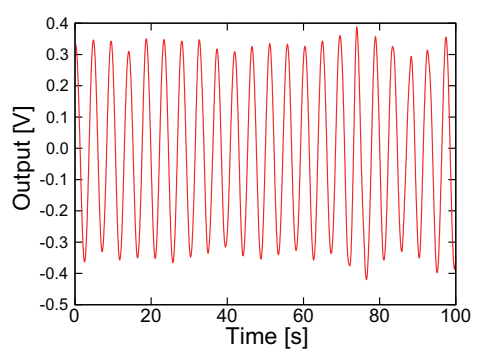

Figure 3. Active spin maser oscillation obtained with the double cell. Shown is the beat signal with a frequency of $0.21 \mathrm{~Hz}$ over a typical period of $100 \mathrm{~s}$, between the maser output and a reference singnal to a lock-in amplifier with a frequency of $33.33 \mathrm{~Hz}$. The temperature was kept at $\sim 100{ }^{\circ} \mathrm{C}$. The determination precision of the average frequency achieved in $10^{5}$ seconds is $4 \mu \mathrm{Hz}$.

amount of ${ }^{129} \mathrm{Xe}$ was increased by a factor of 10 from the single cell used in the development of the ${ }^{3} \mathrm{He}$ comagnetometer [4], in view of the change in the mechanism for optical spin detection. Both the pumping section and the probe section of the cell were cylindrical, $15 \mathrm{~mm}$ in diameter and $10 \mathrm{~mm}$ wide, and they were connected to each other by a tube $5 \mathrm{~mm}$ long and $8 \mathrm{~mm}$ in inner diameter. Only the pumping section was irradiated with the laser light. The FID signal was measured both with the pumping laser light on and off. The result revealed that the amplitudes obtained from both of the two measurements were almost the same, indicating that only the repolarization process is responsible for the optical spin detection in the measurement with the double-cell geometry.

We have succeeded in operating the active spin maser of ${ }^{129} \mathrm{Xe}$ with the double cell, as shown in Fig. 3. Concurrently with this measurement, the FID signal for ${ }^{3} \mathrm{He}$ was also obtained. Here the frequency shift was evaluated as $v\left({ }^{129} \mathrm{Xe}\right)-v\left({ }^{3} \mathrm{He}\right) \cdot \gamma\left({ }^{129} \mathrm{Xe}\right) / \gamma\left({ }^{3} \mathrm{He}\right)$, giving a value of $8 \mathrm{mHz}$, where $v(\mathrm{X})$ and $\gamma(\mathrm{X})$ are the frequency and gyromagnetic ratio of a nucleus $\mathrm{X}$. Thus the frequency shift has been reduced by factor of 10 or more from the case with the single cell, $90 \mathrm{mHz}$ at $100{ }^{\circ} \mathrm{C}$. The residual shift may be attributed to longitudinal repolarization of $\mathrm{Rb}$.

\section{Summary and future perspective}

A double-cell geometry has been introduced into the active nuclear spin maser, in order to reduce the frequency shift due to contact interaction with polarized $\mathrm{Rb}$ valence electrons. The existence of a mechanism other than the transverse repolarization for the optical spin detection had been perceived from our earlier operations of the maser with the single-cell geometry. By taking into account the evaluation from the test, we have succeeded in operating the active spin maser with the double-cell geometry. The frequency shift has been reduced by a factor of 10 or more from the case with the single-cell geometry. The residual shift of about $8 \mathrm{mHz}$ may stem from longitudinal repolarization of $\mathrm{Rb}$. Further reduction may be achieved by shining a linearly polarized light on the masing cell, thus destroying the longitudinal component of $\mathrm{Rb}$ repolarized spins. The validity of this method is already suggested by Fig. 2 (c). Also, the method has another advantage that the drift in the cell temperature, and hence in the Rb number density, can be monitored through the transmission of laser light. [This work was partly supported by the JSPS KAKENHI (No.21104004 and No.21244029).]

\section{References}

[1] M.A. Rosenberry et al., Phys. Rev. Lett. 86, 22 (2001)

[2] A. Yoshimi et al., Phys. Lett. A 304, 13 (2002)

[3] A. Yoshimi et al., Phys. Lett. A 376, 1924 (2012)

[4] Y. Ichikawa et al., Eur. Phys. J. Web of Conferences (this conference) (2013)

[5] Z.L. Ma et al., Phys. Rev. Lett. 106, 193005 (2011)

[6] M.V. Romalis et al., Phys. Rev. A 58, 3004 (1998) 